\title{
Reliability Analysis on Structures Based on a Modified Iterative Response Surface Method
}

\author{
Yanxu Wei $\mathbb{D}^{1},{ }^{1}$ Guangchen Bai, ${ }^{1}$ Bowei Wang, ${ }^{2}$ and Bin Bai $\mathbb{D}^{3}$ \\ ${ }^{1}$ School of Energy and Power Engineering, Beihang University, Beijing 100191, China \\ ${ }^{2}$ School of Computer Science and Engineering, Beihang University, Beijing 100191, China \\ ${ }^{3}$ State Key Laboratory of Reliability and Intelligence of Electrical Equipment, College of Mechanical Engineering, \\ HeBei University of Technology, Tianjin, 300401, China \\ Correspondence should be addressed to Yanxu Wei; weiyanxu2013@163.com
}

Received 5 July 2018; Revised 22 September 2018; Accepted 10 October 2018; Published 25 October 2018

Academic Editor: Marek Lefik

Copyright (C) 2018 Yanxu Wei et al. This is an open access article distributed under the Creative Commons Attribution License, which permits unrestricted use, distribution, and reproduction in any medium, provided the original work is properly cited.

In order to improve the computational accuracy and efficiency of response surface method for reliability analysis on structures, a modified iterative response surface method (called as NDIRSM) is proposed. Firstly, a new starting center point, which is closer to design point, is calculated out as the starting center point instead of the point at mean values of input variables and a dynamic factor vector $\boldsymbol{f}^{1}$, which is inversely proportional to the change rate of performance function with respect to variance, is calculated out for the first iteration. Then the arbitrary factors $f^{k}$ are determined according to the design matrix condition number for the subsequent iteration. Thus the sample points are close to limit state function and the response surface function can approximate the limit state function accurately and efficiently. Two examples are employed to validate the advantages of NDIRSM and the results show that NDIRSM improves the computational accuracy and efficiency of response surface method. At last, NDIRSM is applied to the reliability analysis on low cycle fatigue life of a gas turbine disc, which provides a useful reference for reliability analysis on low cycle fatigue life of gas turbine disc and demonstrates the high computational accuracy and efficiency of NDIRSM.

\section{Introduction}

There are many uncertain variables, such as geometry parameters, loadings, and material properties, which influence the safety of structures, so the estimation of the reliability probability of structures is very important. However, the performance function $(\mathrm{PF})$ is implicit for the most of practical engineering structures, so it is very difficult to estimate the reliability probability of structures with analytic methods. So in order to calculate the reliability probability of structures, many methods are proposed. Monte Carlo simulation [1] (MCS) provides an accurate method to predict failure probability, but it is very time consuming because it needs too many finite element executions, especially for the complex structures with low failure probabilities. The first-order reliability method (FORM) and the second-order reliability method (SORM) [2-5] are difficult to apply to the structure reliability analysis because the PF of structures generally cannot be expressed explicitly. Response surface method (RSM) is an appropriate method to estimate the failure probability, which approximates the limit state function (LSF), which is defined as $P F=0$, with a simple and explicit function with respect to random variables such as a quadratic polynomial. It needs a few finite element executions and provides an accurate result relatively. So it has been studied by many researchers.

Bucher and Bourgund [6] suggested an iterative RSM with an adaptive interpolation scheme to improve the accuracy of RSM. The response surface function (RSF) is a quadratic polynomial without cross terms to improve the efficiency. Then the Monte Carlo simulation (MCS) is employed to obtain the failure reliability. Based on this, Rajashekhar and Ellingwood [7] proposed an adaptive iterative RSM to improve the computational accuracy which more iteration is needed until the convergent condition is achieved.

Kim and $\mathrm{Na}$ [8] used the gradient projection method to select the sample points so that the sample points are close to LSF, which improve the efficiency and accuracy of 
RSM. Taking into account the knowledge of the engineering, Gayton et al. [9] proposed an improved RSM. A resampling technique, which is called Complete Quadratic Response Surface with Resampling (CQ2RS), is used and a confidence interval is used to check the result. In order to make full use of the sample points close to the LSF, Kaymaz and McMahon [10] proposed an improved RSM, which normal regression is replaced by a weighted regression, so that the sample points closer to the LSF can have larger weighted factors when calculating the coefficients of the RSF. Xuan Son Nguyen et al. [11] proposed an adaptive RSM based on a double weighted regression technique, which can improve the convergence speed and reduce the computational effort. Soo-Chang Kang et al. [12] propose a RSM based on the moving least squares approximation. In order to reduce the computational efforts, a linear polynomial is used in the first iteration and a quadratic polynomial without mixed terms is employed for the subsequent iteration. Allix and Carbone [13] proposed an improved RSM. The sample points are selected after rotating the coordinate system according to the sensitivity vector of the LSF in U-space, which improves the accuracy without increasing the computational efforts. Guan and Melchers [14] studied on the effect of different values of the arbitrary factor $f^{k}$ ( $k$ represents the $k t h$ iteration). It is observed in the paper that the arbitrary factor $f^{k}$ has a considerable effect on approximating the LSF and the shape of the LSF has a great effect on it. The author suggests that the appropriate value of the arbitrary factor $f^{k}$ is around 2 or 3. Gavin and Yau [15] research on the use of high-order polynomials as RSF. The order of the polynomial is determined through a statistical analysis of polynomial coefficients. Compared with the second-order RSM, more accurate results are reached. Roussouly et al. [16] proposed an improved RSM that the most important variables are selected according to the statistical criteria and cross-validation method with Latin Hypercube Sampling (LSH) method firstly. Then LHS is refined in a region of interest defined with respect to an important level on probability density in the design point. Finally, Bootstrapbased indicators are adopted to evaluate the influence of the error on the failure probability. Gaspar et al. [17] proposed an efficient RSM with the Krigng interpolation models instead of the quadratic polynomial without cross terms. Goswami et al. [18] proposed an improved RSM with the moving leastsquares method, which can reduce the number of iteration. Shayanfar et al. [19] proposed a new efficient RSM, which is combined with the importance sampling concepts. The vicinity of the design point is located with the method of the concepts of importance sampling and an effective twostep updating role, through which a suitable seedbed for the subsequent iteration of RSM is obtained. Then the RSM, with Bucher experimental design, starts to work from the seedbed. Ali Hadidi et al. [20] proposed an improved RSM with the exponential RSF instead of quadratic polynomial without cross terms. The sample points are selected through a novel approach and an experimental updating technique is applied. The accuracy and efficiency are improved through this method.
In order to improve the computational accuracy and efficiency of RSM, RSM is also studied with some intelligent algorithms, such as the neural network [21-23] and the support vector machines [24-26].

In the process of RSM, it can be concluded that the positions of sample points are very important since the result of RSM is determined by them to a great extent. And the positions of sample points in each iteration is up to the sample points for the first iteration and the arbitrary factors $f^{k}$ ( $k$ represents the $k t h$ iteration) for the subsequent iteration, so it is very important to get appropriate sample points for the first iteration and the arbitrary factors $f^{k}$ for the subsequent iteration. In this paper, a new starting center point is calculated out as the starting center point which is close to design point for the first iteration and, in order to make the surrounding sample points close to the design point, a dynamic factor vector $f^{1}$ is proposed for the first iteration. Then reasonable arbitrary factors $f^{k}$ for the subsequent iteration are selected according to the design matrix condition number. Based on these, a modified iterative response surface method (called as NDIRSM) is proposed.

In what follows, Section 2 introduces the classical iterative RSM. Based on this, Section 3 modifies the conventional iterative RSM with a new starting center point and dynamic factor vector $f^{1}$ for the first iteration and reasonable arbitrary factors $f^{k}$ for the subsequent iteration are determined according to the design matrix condition number. Section 4 validates NDIRSM with two examples. Section 5 discusses reliability analysis on low cycle fatigue life of gas turbine disc with NDIRSM. Section 6 summaries the conclusions.

\section{The Classical Iterative Response Surface Method}

In classical iterative RSM, in order to reduce the number of sample points and improve the efficiency, a quadratic polynomial without cross terms is employed by Bucher and Bourgund [6] as the RSF, so only $2 n+1$ sample points are needed in each iteration. The quadratic polynomial without cross terms can be expressed as

$$
\bar{g}(\boldsymbol{x})=a+\sum_{i=1}^{n} b_{i} x_{i}+\sum_{i=1}^{n} c_{i} x_{i}^{2}
$$

where $\bar{g}(\boldsymbol{x})$ is RSF and $a, b_{i}$, and $c_{i}$ are the coefficients of polynomials which represent the RSF; $n$ denotes the number of random variables.

The values of the coefficients in (1) are evaluated by $2 n+1$ sample points and their PF values, so the positions of the $2 n+1$ sample points are very important. The $2 n+1$ sample points for the first iteration are starting center point (SCP) $\boldsymbol{x}_{\mathrm{c}}$, which is a point at the mean value $x_{\mathrm{m}}$ of the input random variables $\boldsymbol{x}=\left[x_{1}, x_{2} \ldots x_{n}\right]$, and the surrounding sample points $\boldsymbol{x}_{\mathrm{c}} \pm$ $f^{k} \boldsymbol{\sigma}$. Generally, the value of arbitrary factor $f^{\mathrm{k}}$ is 3 in classical RSM. The coefficients of (1) are evaluated by

$$
\boldsymbol{b}=\boldsymbol{x}^{-1} \boldsymbol{g}
$$


where $\boldsymbol{b}$ is the vector of the coefficients of RSF; $\boldsymbol{g}$ is the vector of the corresponding values of PF; $\boldsymbol{x}$ is the design matrix, which is expressed as follows when a quadratic polynomial without mixed terms is used.

$$
\boldsymbol{x}=\left[\begin{array}{ccccccc}
1 & x_{11} & \cdots & x_{1 n} & x_{11}^{2} & \cdots & x_{1 n}^{2} \\
1 & x_{21} & \cdots & x_{2 n} & x_{21}^{2} & \cdots & x_{1 n}^{2} \\
\vdots & \vdots & \cdots & \vdots & \vdots & \cdots & \vdots \\
1 & x_{n 1} & \cdots & x_{n n} & x_{n 1}^{2} & \cdots & x_{n n}^{2}
\end{array}\right]
$$

Then the FORM is used to obtain the reliability index $\beta$ and design point $\boldsymbol{x}_{\mathrm{d}}$.

A linear interpolation proposed by Bucher and Bourgund [6] (given by (4)) is used to generate a new center point $\boldsymbol{x}_{\mathrm{c}}$ for the next iteration, which is expressed as

$$
\boldsymbol{x}_{\mathrm{c}}=\boldsymbol{x}_{\mathrm{m}}+\left(\boldsymbol{x}_{\mathrm{d}}-\boldsymbol{x}_{\mathrm{m}}\right) \frac{g_{\mathrm{m}}}{g_{\mathrm{m}}-g\left(\boldsymbol{x}_{\mathrm{d}}\right)}
$$

Equation (4) ensures that the new center point $\boldsymbol{x}_{\mathrm{c}}$ is closer to LSF than design point $\boldsymbol{x}_{\mathrm{d}}$ obtained in previous iteration.

The process above is repeated until an accurate result is obtained.

\section{The Improved Response Surface Method}

During to the process of classical RSM, it can be seen that the key points of RSM are the positions of sample points for first iteration and arbitrary factors $f^{k}$ after the form of the RSF is selected. In order to improve the computational accuracy and efficiency of RSM, a new method of selecting the sample points for first iteration is proposed. Then the arbitrary factors $f^{k}$ are calculated out according to the design matrix condition number of (3). Based on these, NDIRSM is proposed.

3.1. The New Starting Center Point. To improve the computational efficiency and accuracy, a new starting center point (NSCP) is proposed to replace the SCP in this paper, which is closer to the design point $\boldsymbol{x}_{\mathrm{d}}$ than the SCP at the mean values $\boldsymbol{x}_{\mathrm{m}}$ of random variables. In order to get the NSCP, a final direction $\boldsymbol{u}$ is constructed, which is approximately pointing at the design point from the point at mean values in standard normal space.

Firstly, the point at the mean values $\boldsymbol{x}_{\mathrm{m}}$ and $n$ surrounding sample points $\boldsymbol{x}_{\mathrm{m}}-f \boldsymbol{\sigma}$ are selected and the corresponding values of $\mathrm{PF} g_{i}$ are calculated out. The value of $f$ is 1-3 in some literatures $[7,8,10,12-14,16]$ and it is advised that the value of $f$ is 1.5 since it is used to obtain the basic information of PF around SCP. Based on the basic information of PF, the change rate vector $\boldsymbol{\eta}$ around $\boldsymbol{x}_{\mathrm{m}}$ is defined as

$$
\eta_{i}=\frac{g_{i}-g_{\mathrm{m}}}{f \sigma_{i}}=r_{i}\left(\sigma_{i}^{-1}\right) \quad i=1,2,3, \cdots, n ;
$$

The components of the vector $\boldsymbol{r}$ in (5) are normalized with the expression as follows:

$$
p_{i}=\frac{r_{i}}{\max \left(\left|r_{j}\right|\right)} \quad i=1,2,3, \cdots, n ; j=1,2,3, \cdots, n
$$

It can be seen that the value of $p_{i}$ is between -1 and 1 .

The absolute value of $p_{i}$ indicates the influence of $\sigma_{i}$ on the value of PF around the mean values $\boldsymbol{x}_{\mathrm{m}}$. If the absolute value of $p_{i}$ is larger, it means that $\sigma_{i}$ has a greater influence on the value of PF; to the contrary, if the absolute value of $p_{i}$ is smaller, it means that $\sigma_{i}$ has a less influence on the value of PF. Regarding the sign of $p_{i}$, if the sign of $p_{i}$ is negative, it indicates that $-f \sigma_{i}$ have a negative influence on the value of PF while if the sign of $p_{i}$ is positive, it indicates that $-f \sigma_{i}$ have a positive influence on the value of PF. Based on these, the final direction $\boldsymbol{u}$ is defined according to the influence of $p_{i}$ on the value of PF firstly, which is expressed as

$$
u_{i}= \begin{cases}0.25 \times \operatorname{sign}\left(p_{i} g_{\mathrm{m}}\right) & 0 \leq\left|p_{i}\right|<0.3 \\ 0.5 \times \operatorname{sign}\left(p_{i} g_{\mathrm{m}}\right) & 0.3 \leq\left|p_{i}\right|<0.6 \\ 1 \times \operatorname{sign}\left(p_{i} g_{\mathrm{m}}\right) & 0.6 \leq\left|p_{i}\right| \leq 1 \\ & i=1,2,3, \cdots, n\end{cases}
$$

where $\operatorname{sign}\left(p_{i} g_{\mathrm{m}}\right)$ is a sign function, which is expressed as

$$
\operatorname{sign}\left(p_{i} g_{\mathrm{m}}\right)=\left\{\begin{array}{ll}
-1 & p_{i} g_{\mathrm{m}}<0 \\
1 & p_{i} g_{\mathrm{m}}>0 \\
0 & p_{i} g_{\mathrm{m}}=0
\end{array} \quad i=1,2,3, \cdots, n\right.
$$

Equation (8) ensures that the final direction points to LSF in standard normal space no mater $g_{\mathrm{m}}$ is greater or less than zero. The vector obtained with (8) is called as initial direction vector $\boldsymbol{v}$.

The coefficients before $\operatorname{sign}\left(p_{i} g_{\mathrm{m}}\right)$ in $(7)$ are the weight coefficients, which are selected between 0 and 1 according to the absolute value of $p_{i}$, so the coefficients represent the influence of $\sigma_{i}$ on the value of PF. Though the coefficients can be selected by others to represent the influence of $\sigma_{i}$ on the value of PF, the values of coefficients in (7) are accuracy enough for the proposed method. The coefficients after $\operatorname{sign}\left(p_{i} g_{\mathrm{m}}\right)$ in (7) are used to divided the range of $\boldsymbol{p}$ into corresponding number of approximately equal intervals.

It can be concluded from many examples in many literatures $[7,8,10,12-14,16]$ that the components of exact direction vector $\left(\boldsymbol{x}_{\mathrm{d}}-\boldsymbol{x}_{\mathrm{m}}\right) \cdot / \boldsymbol{\sigma} \boldsymbol{e d}$ (pointing at the design point in standard normal coordinates) have positive correlations with the components of vector $\boldsymbol{p}$, and the direction of exact direction vector $\boldsymbol{e} \boldsymbol{d}$ and the final direction $\boldsymbol{u}$ are located closely, which is also demonstrated with the examples in Sections 4 and 5. So the NSCP, which is close to LSF, obtained along the final direction is close to design point.

However, for many problems in many literatures $[7,8$, $10,12-14,16]$, the biggest value of the components of exact direction vector $\left(\boldsymbol{x}_{\mathrm{d}}-\boldsymbol{x}_{\mathrm{m}}\right) . / \boldsymbol{\sigma} \boldsymbol{e d}$ is around 2 , so 2 is selected as the biggest coefficients before $\operatorname{sign}\left(p_{i} g_{\mathrm{m}}\right)$ in (7), so the coefficients before $\operatorname{sign}\left(p_{i} g_{\mathrm{m}}\right)$ in (7) are multiplied by 2 and (7) becomes the following: 


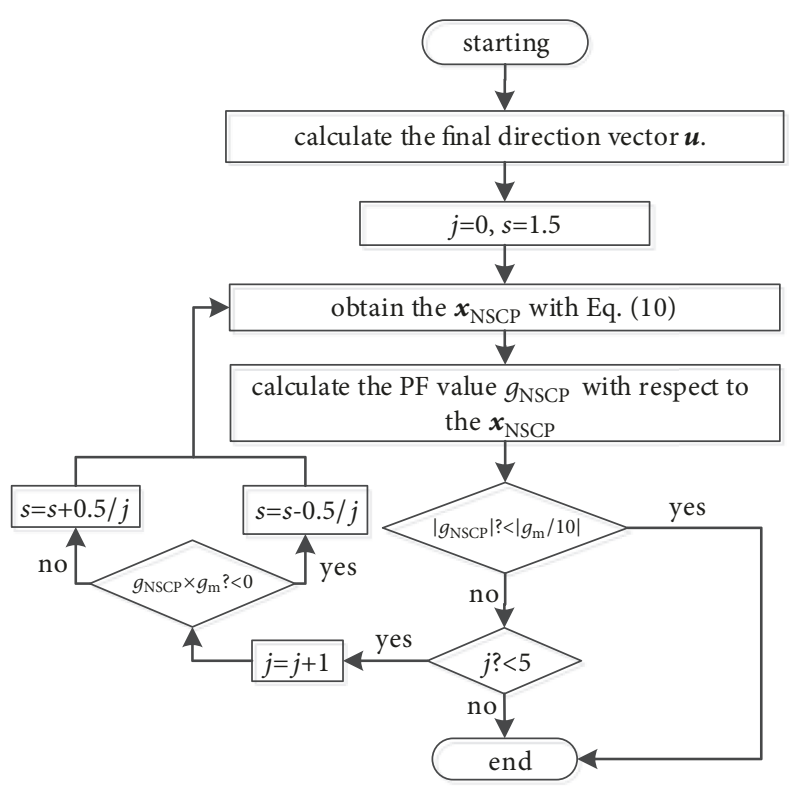

Figure 1: Process of obtaining the coefficient $s$.

$$
u_{i}= \begin{cases}0.5 \times \operatorname{sign}\left(p_{i} g_{\mathrm{m}}\right) & 0 \leq\left|p_{i}\right|<0.3 \\ 1 \times \operatorname{sign}\left(p_{i} g_{\mathrm{m}}\right) & 0.3 \leq\left|p_{i}\right|<0.6 \\ 2 \times \operatorname{sign}\left(p_{i} g_{\mathrm{m}}\right) & 0.6 \leq\left|p_{i}\right| \leq 1 \\ & \quad i=1,2,3, \cdots, n\end{cases}
$$

Then the final direction $\boldsymbol{u}$ is obtained. with

At last, the NSCP is sought for along the final direction

$$
\begin{aligned}
\boldsymbol{x}_{\mathrm{NSCP}} & =\boldsymbol{x}_{\mathrm{m}}+s \times \boldsymbol{u} . \times \boldsymbol{\sigma} \\
& =\boldsymbol{x}_{\mathrm{m}}+s \times\left(u_{1} \sigma_{1}, u_{2} \sigma_{2}, \cdots, u_{n} \sigma_{n}\right)
\end{aligned}
$$

where.$\times$ means the multiplication of the corresponding between two vector as shown in Eq. (10). $s$ is a coefficient. For $g_{\mathrm{m}}>0$, if the value of $s$ becomes large, the PF value $g_{\mathrm{NSCP}}$ of $\boldsymbol{x}_{\mathrm{NSCP}}$ will become small; if the value of $s$ becomes small, the PF value $g_{\mathrm{NSCP}}$ of $\boldsymbol{x}_{\mathrm{NSCP}}$ will become large. For $g_{\mathrm{m}}<0$, if the value of $s$ becomes small, the PF value $g_{\mathrm{NSCP}}$ of $\boldsymbol{x}_{\mathrm{NSCP}}$ will become small; if the value of $s$ becomes large, the PF value $g_{\mathrm{NSCP}}$ of $\boldsymbol{x}_{\mathrm{NSCP}}$ will become large. So the PF value $\boldsymbol{x}_{\mathrm{NSCP}}$ can be close to zero along the final direction through changing the value of $s$. The criterion close to zero is that the absolute value of $g_{\mathrm{NSCP}}$ is less than $g_{\mathrm{m}} / 10$ and the value of $g_{\mathrm{m}} / 10$ is a compromise of the efficiency and accuracy. The details of searching for NSCP are shown in Figure 1.

The process of obtaining the final direction and $\boldsymbol{x}_{\mathrm{NSCP}}$ is shown in Figure 2. The initial direction vector obtained by (8) constructs a direction that is pointing to the LSF. The final direction vector $\boldsymbol{u}$ obtained by (9) forms a direction that is approximately pointing at design point. The exact direction in Figure 2 is pointing at design point. It can be seen from Figure 2 that the final direction and the exact direction are closely located compared with the initial direction and the

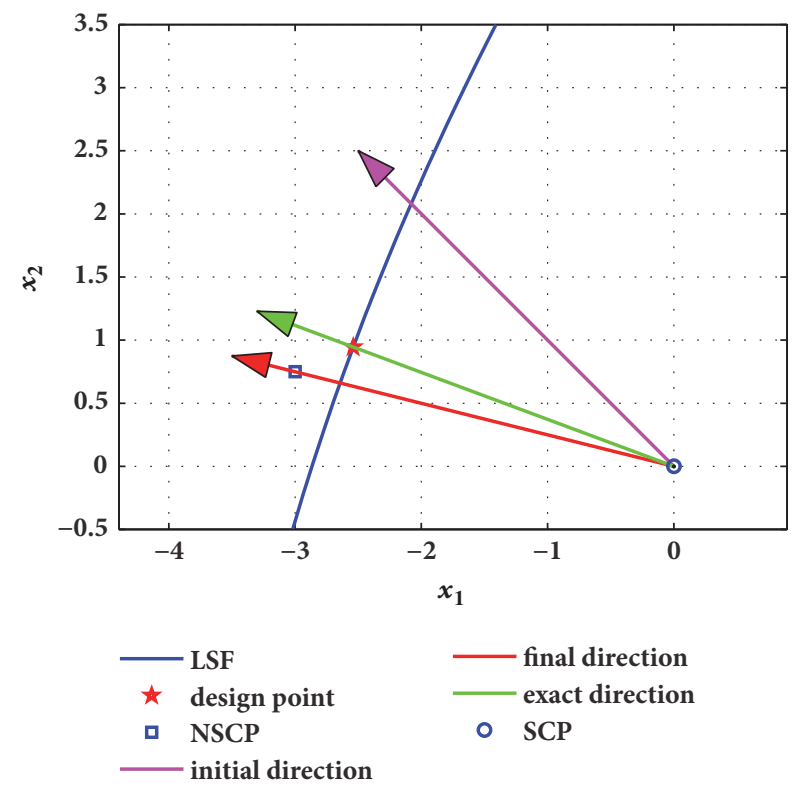

FIGURE 2: Process of obtain the final direction and NSCP.

NSCP, which is in the final direction, is close to the design point.

3.2. The Value of the Arbitrary Factor $f^{k}$. The arbitrary factor $f^{k}$ can affect the approximation of LSF since the positions of surrounding sample points are determined by it after the center point is determined. If the arbitrary factor $f^{k}$ is too small, the sample points only have an effect on a small region and even (2) to evaluate coefficients of the RSF becomes illconditioned. If the arbitrary factor $f^{k}$ is too large, the details between the sample points may be neglected, and even a wrong result may be calculated out when the LSF is highly nonlinear around the design point. So an appropriate value of the arbitrary factor $f^{k}$ is very important. And as stated in Section 3.1, it is advised that the value of $f$ is 1-3 in some literatures $[7,8,10,12-14,16]$. The value of arbitrary factor $f^{k}$ should be large firstly so that the algorithm can expend the searching region. After a center point close to the design point is found, the value of arbitrary factor $f^{k}$ should be small to obtain the details of LSF around the design point. Based on these, a new method of selecting the arbitrary factor $f^{k}$ is proposed.

3.2.1. Dynamic Factor Vector $\boldsymbol{f}^{1}$ for the First Iteration. If the PF value of sample point $\boldsymbol{x}$ is close to zero, it indicates that the sample point $\boldsymbol{x}$ is close to LSF; to the contrary, it indicates that the sample point $\boldsymbol{x}$ is away from LSF. The PF value $g_{\mathrm{NSCP}}$ of $\boldsymbol{x}_{\mathrm{NSCP}}$ is close to zero so the NSCP is very close to LSF. In order to make the sample points around the NSCP close to LSF, $f_{i}^{1}$ should be inversely proportional to the change rate of LSF with respect to $\sigma_{i}$. However, the change rate of LSF around the $\boldsymbol{x}_{\mathrm{NSCP}}$ cannot be obtained, so a change rate defined in (5) of PF around the $\boldsymbol{x}_{\mathrm{m}}$ is employed to approximately substitute the change rate of LSF around the $\boldsymbol{x}_{\mathrm{NSCP}}$, and the parameter obtaining the factor $f_{i}^{1}$ is expressed as 


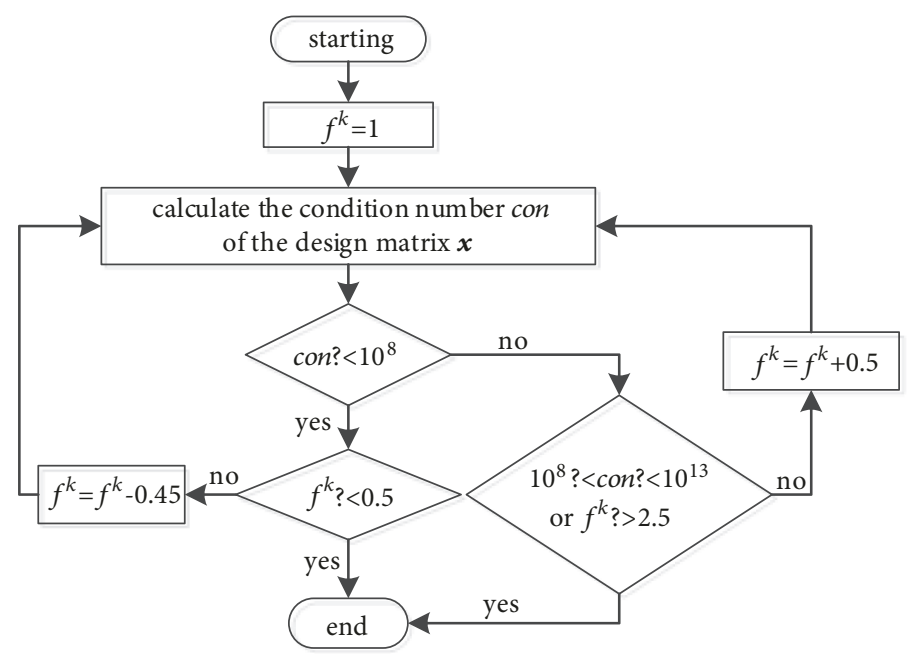

FIGURE 3: Process of calculating the factor $f^{k}$.

$$
\lambda_{i}=\frac{\min \left(\left|r_{j}\right|\right)}{\left|r_{i}\right|} \quad i=1,2,3, \cdots, n ; j=1,2,3, \cdots, n
$$

$r_{i}$ is the change rate of $\mathrm{PF}$ with respect to $\sigma_{i}$ as shown in (5). It can be seen that $0<\lambda_{i} \leq 1$. Then in order to make the value of $f$ in the range of 1-3, an exponential function is employed expressed as

$$
f_{i}^{1}=3^{\lambda_{i}} \quad i=1,2,3, \cdots, n
$$

Then the surrounding sample points can be obtained through $\boldsymbol{x}_{\mathrm{m}} \pm f_{i}^{1} \boldsymbol{\sigma}$, which are close to LSF. The surrounding sample points obtained with dynamic factor vector are shown in Figures 6 and 9.

3.2.2. Factors $f^{k}$ for the Subsequent Iteration. In order to evaluate the reliability index accurately, the arbitrary factor $f^{k}$ should be as small as possible on the condition of the design matrix condition number of (3) not being illconditioned, when the center point is close enough to the design point. After the calculation of the first iteration, the center point is very close to the design point, so the arbitrary factor $f^{k}$ should be as small as possible on the condition of the design matrix condition number of (3) not being illconditioned. In order to select appropriate values of arbitrary factor $f^{k}$ for the subsequent iteration, a method according to the design matrix condition number of (3) is proposed.

The details of the method are shown in Figure 3. It can be seen from Figure 3 that the least value of factor $f^{k}$ obtained with this method is 0.1 and the largest value of factor $f^{k}$ obtained with this method is 3 . If the condition number of design matrix is small enough, the algorithm decreases the factor $f^{k}$ to improve the computational accuracy and efficiency of RSM; if the condition number of design matrix is too large, it can be decreased through increasing the value of factor $f^{k}$. Thus the algorithm can obtain a factor $f^{k}$ as small as possible on the condition of the design matrix condition number not being ill-conditioned, and the RSF can approximate the LSF accurately around the design point.
3.3. The Procedure of NDIRSM. To improve the computational accuracy and efficiency of RSM, the NSCP and dynamic factor vector are proposed for the first iteration. The sample points generated with the NSCP and dynamic factor vector are close to LSF around the design point, so an accurate reliability index $\beta$ can be obtained after the first iteration. Then the RSF is established with a quadratic polynomial without cross terms. Reliability index $\beta$ and design point $\boldsymbol{x}_{\mathrm{d}}$ are calculated out through FORM. For the subsequent iteration, the design point $\boldsymbol{x}_{\mathrm{d}}$ is selected as the center point for the next iteration and an appropriate factor $f^{k}$ is obtained with the method in Section 3.2.2, which can improve computational accuracy and efficiency.

The result of reliability analysis of RSM is reliability index $\beta$, so the algorithm can be stopped when reliability index $\beta$ satisfies a certain precision and the stopping criterion can be expressed as

$$
\frac{\beta^{k}-\beta^{k-1}}{\beta^{k}}<\varepsilon
$$

where $\beta$ is the reliability index and $k$ indicates the $k$ th iteration. $\varepsilon$ is equal to 0.001 . Then NDIRSM is formed, the process of which is shown in Figure 4.

\section{Verification of NDIRSM with Testing Examples}

In this section, two mathematical problems are used to demonstrate the computational accuracy and efficiency of NDIRSM.

4.1. Example One: An Exponential Function. The first example is an exponential function, expressed as

$$
\begin{aligned}
g(\boldsymbol{x})= & \exp \left[0.4\left(x_{1}+2\right)+6.2\right]-\exp \left(0.3 x_{2}+5.0\right) \\
& -200
\end{aligned}
$$

where $x_{1}$ and $x_{2}$ are independent variables and have a standard normal distribution, respectively. This example is 


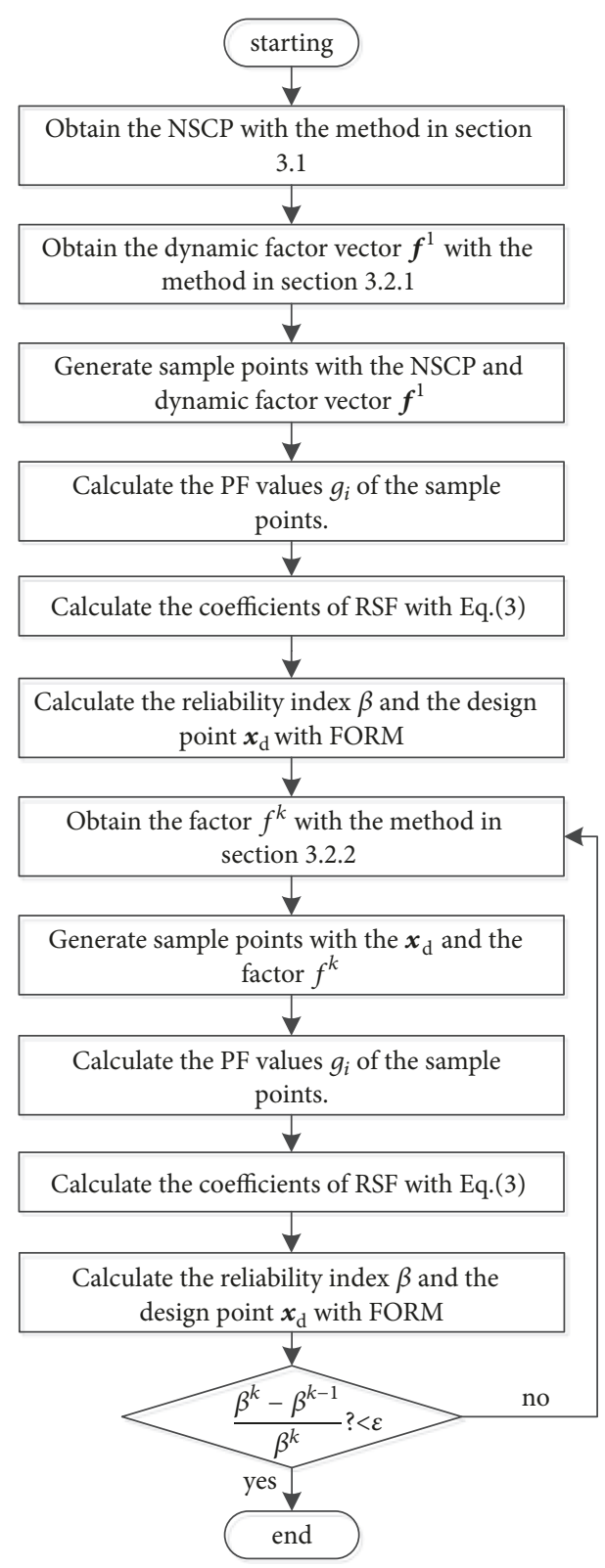

FIgURE 4: Process of NDIRSM.

studied by many researchers $[11,12,16,27,28]$. Some of the results are shown in Table 1.

According to NDIRSM proposed in this paper, the initial sample points for calculating the NSCP are $\boldsymbol{x}_{\mathrm{m}}\left(\begin{array}{ll}0 & 0), \boldsymbol{x}_{1}\end{array}\right.$ $(0-1.5)$ and $x_{2}(-1.50)$ and the corresponding values of PF are $748.220,254.432$ and 802.000 . Based on these, the initial direction vector $\boldsymbol{v}$, which is [-1 1$]$, is calculated out according to $(8)$ and the final direction vector, which is $[-20.5]$, is calculated out by (9). Finally, the NSCP is obtained with the method in Section 3.1.

It can be seen from Figure 5 that the initial direction $v$ is a direction along which the value of $\mathrm{PF}$ is close to zero and the final direction $\boldsymbol{u}$ is approximately pointing at the design point. The final direction $\boldsymbol{u}$ and the exact direction $\boldsymbol{e d}$ are located closely compared with the initial direction. Figures 5 and 6

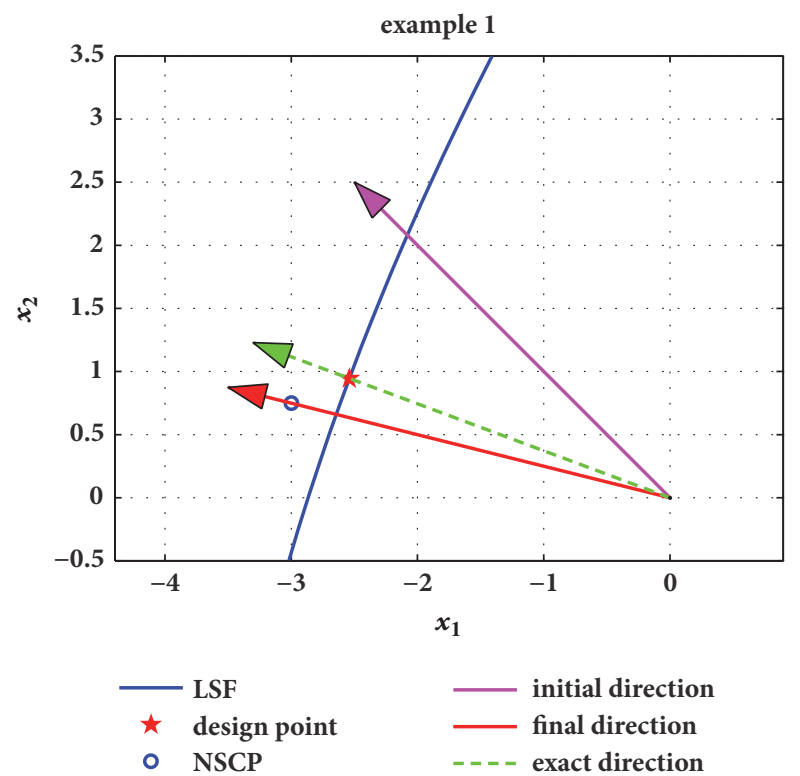

FIGURE 5: The new starting center point and directions.

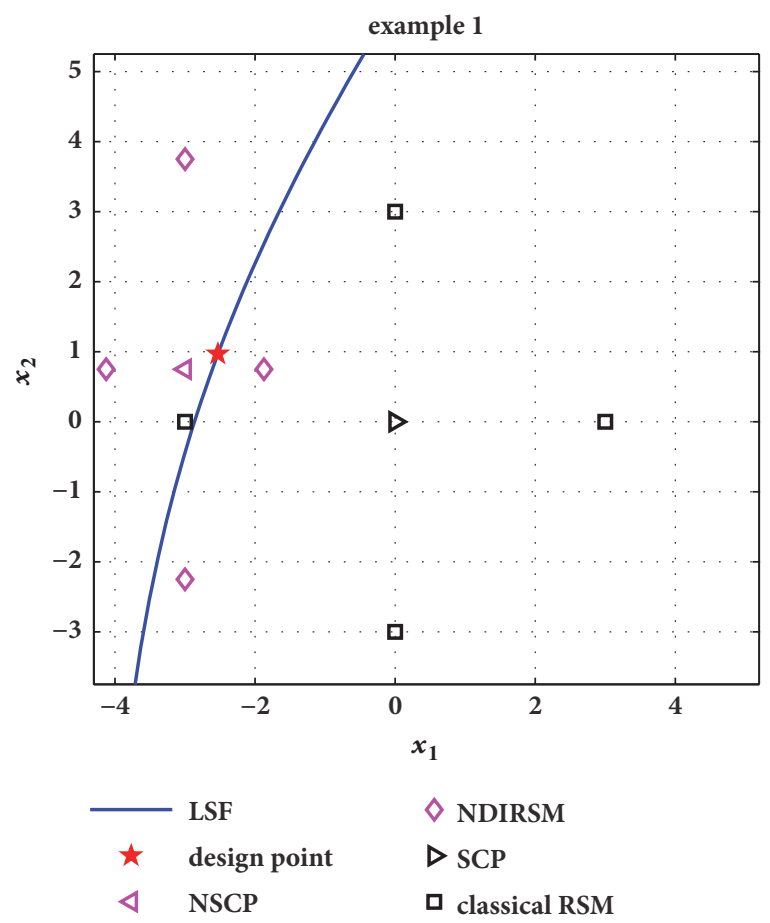

FIgURE 6: Sample points for the first iteration.

show that the NSCP is very close to the design point, which is easier to find the design point than SCP at the mean values $\boldsymbol{x}_{\mathrm{m}}$.

The appropriate factor $f^{k}$ can be obtained with the method in Section 3.2 for each iteration. The dynamic factor vector $f^{1}$ obtained by the method in Section 3.2.1 for the first iteration is [1.1268 3.0000] and the surrounding sample points obtained with $f^{1}$ are shown in Figure 6, from which it can be seen that the sample points are closer to LSF compared 
TABLE 1: Results of example 1.

\begin{tabular}{lcccc}
\hline method & $\beta$ & $x_{1}$ & $x_{2}$ & $\begin{array}{c}\text { Number of sample } \\
\text { points }\end{array}$ \\
\hline FORM [12] & 2.7099 & -2.5398 & 0.9450 & 27 \\
NDIRSM & 2.7099 & -2.5398 & 0.9449 & 13 \\
Method in [11] & 2.707 & -2.567 & 0.860 & 12 \\
Method in [12] & 2.7100 & -2.5411 & 0.9417 & 12 \\
Method in [27] & 2.7099 & -2.5478 & 0.9236 & Not avail. \\
Method in [28] & 2.7103 & -2.538 & 0.951 & 21 \\
\hline
\end{tabular}

TABLE 2: Successive results of example 1 with different methods.

\begin{tabular}{|c|c|c|c|c|c|}
\hline \multirow{2}{*}{ items } & \multirow{2}{*}{$\beta$ (NDIRSM) } & \multicolumn{4}{|c|}{$\beta($ classical RSM $)$} \\
\hline & & $f^{k}=3$ & $f^{k}=2$ & $f^{k}=1$ & $f^{k}=0.1$ \\
\hline First iteration & 2.7093 & 2.1222 & 2.8610 & 2.5132 & 1.5308 \\
\hline Final result & 2.7099 & 2.7113 & 2.7102 & 2.7099 & 2.7099 \\
\hline Number of sample points & 13 & 29 & 23 & 17 & 17 \\
\hline Number of iteration & 2 & 5 & 4 & 3 & 3 \\
\hline$x_{1}$ & -2.5398 & -2.5725 & -2.555 & -2.5437 & -2.5397 \\
\hline$x_{2}$ & 0.9449 & 0.8566 & 0.9033 & 0.9344 & 0.9453 \\
\hline
\end{tabular}

with classical RSM. For this example, the center point for second iteration is almost the same place as the design point, which is shown in Figure 7. So the value of $f^{2}$ obtained with the method in Section 3.2 is relatively small and the details around the design point is obtained, which can improve the computational accuracy and efficiency of RSM.

The final result with NDIRSM proposed in this paper is shown in Table 1 . The algorithm is converged in 2 iterations with 13 sample points. The reliability index in this paper is calculated out with FORM, so the result of FORM is considered as the exact result. In terms of the reliability index $\beta$ and design point $\boldsymbol{x}_{\mathrm{d}}$, it can be seen from Table 1 that the NDIRSM proposed in this paper can achieve a more accurate solution with almost the same number of sample point that represents the efficiency of algorithm.

The processes of iteration are listed in Table 2 including the NDIRSM proposed in this paper and classical RSM with different $f^{k}$. The NDIRSM is converged after two iterations, which is fewer than classical RSM. Compared with the classical RSM, an accurate reliability index in the first iteration is obtained through NDIRSM, which lead to a fewer iteration. Compared with the exact result listed in Table 1, a more accurate result is obtained by NSIRSM in terms of the design point and reliability index.

It can be seen from Table 2 that it needs only one more iteration for the classical RSM with $f^{k}=0.1$ compared with NDIRSM. That is, only 4 more sample points are required to get almost the same result. However, there are many input variables for practical engineering problems and more sample points are required for each iteration with classical RSM. So the NDIRSM can improve the efficiency of RSM significantly for the problems with many input variables.

The final direction vector $\boldsymbol{u}$ of this example is $[-2$, 0.5]. The exact direction vector $\left(\boldsymbol{x}_{\mathrm{d}}-\boldsymbol{x}_{\mathrm{m}}\right) \cdot / \boldsymbol{\sigma}$ ed is

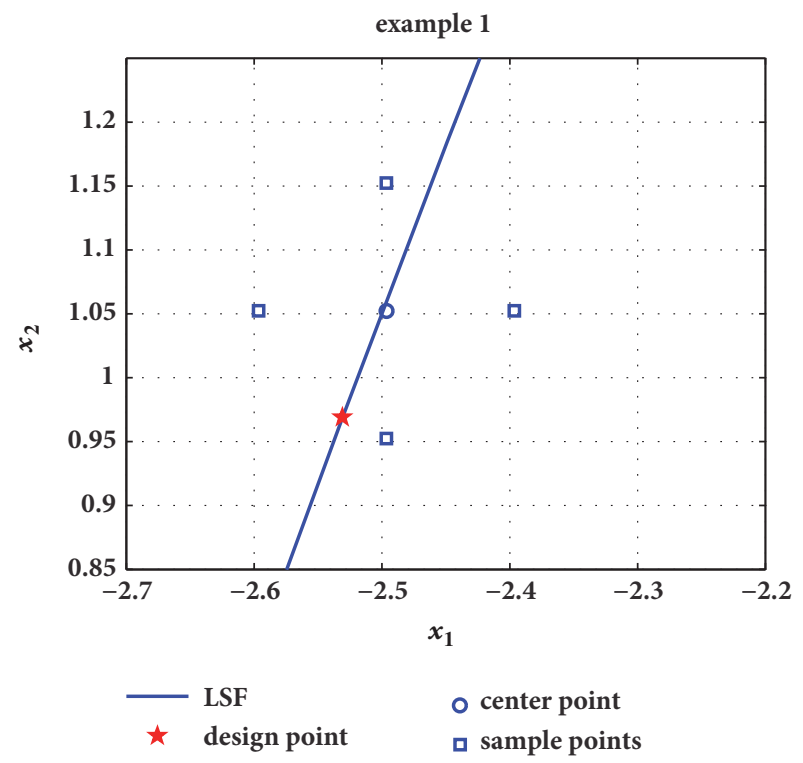

Figure 7: Sample points for the second iteration.

$[-2.5398,0.9450]$, which is obtained with the reference result listed in Table 1 . Then the ed is transformed with the equation $e d_{i}=e d_{i} / \max (|e d|) \times 2$ to keep the biggest value of $e d_{i}$ equal to the biggest value of $u_{i}$ and the result is $[-2,0.744]$. It can be seen that the values of the corresponding components $u_{i}$ and $e d_{i}$ are equal to or close to each other, which indicates that the directions they represent are close to each other as shown in Figure 5.

4.2. Example Two: A Cantilever Beam. This example is a cantilever beam with a rectangular cross, which is subjected to uniformly distributed loading. The maximum deflection 
TABLE 3: Results of example 2.

\begin{tabular}{lcccc}
\hline method & $\beta$ & $x_{1}$ & $x_{2}$ & $\begin{array}{c}\text { Number of sample } \\
\text { points }\end{array}$ \\
\hline FORM [20] & 2.3309 & 0.588 & -2.256 & 30 \\
NDIRSM & 2.3309 & 0.5860 & -2.2560 & 21 \\
Method in [9] & 2.318 & 0.586 & -2.243 & 24 \\
improved RSM $\left(f_{i}=1\right)[13]$ & 2.33 & 0.595 & -2.258 & 50 \\
proposed iRSM [20] & 2.3310 & 0.580 & -2.258 & 30 \\
Method in [27] & 2.3309 & 0.5946 & -2.2530 & Not avail. \\
Method in [28] & 2.3302 & 0.595 & -2.253 & 19 \\
\hline
\end{tabular}

is greater than $l / 325$. The performance function is expressed as:

$$
g=\frac{(w \times b) l^{4}}{8 E I}+\frac{l}{325}
$$

Where $w$ denotes the load per unit area; $b$ and $l$ denotes the width and length of the beam, respectively; $E$ is the elastic modulus and $I$ is the moment of inertia of the cross section. $E$ and $l$ equal to $2.6 \times 10^{4} \mathrm{MPa}$ and $6 \mathrm{~m}$, respectively, as deterministic parameters. $x_{1}$ and $x_{2}$ represent the random values of the load $w$ and the height $h$, with $x_{1} \sim N\left(0.001,0.0002^{2}\right)$ and $x_{2} \sim N\left(250.0,37.5^{2}\right)$. Then the following function is obtained.

$$
g(\boldsymbol{x})=18.46154-7.476923 \times 10^{10} \frac{x_{1}}{x_{2}^{3}}
$$

This example is a polynomial with division, which is studied by many researchers $[9,13,20,27,28]$. Some of the results are shown in Table 3.

In order to calculate the NSCP, the point at the mean values $[0.001,250.0]$ and 2 surrounding sample points $x_{\mathrm{m}}-$ $f \sigma$, which are $[0.0007,250]$ and $[0.001,193.75]$, are selected. The corresponding values of PF are 13.6763, 15.1119 and 8.1814. The initial direction vector $\boldsymbol{v}$, which is $[1,-1]$, is calculated out with (8). Then the final direction vector, which is $[0.5,-2.0]$, is calculated out by (9). Finally, the NSCP is obtained with the method in Section 3.1.

It can be seen from Figure 8 that the initial direction $v$ is a direction along which the value of PF is close to zero, and the final direction $\boldsymbol{u}$ is approximately pointing at the design point. The final direction $\boldsymbol{u}$ and the exact direction $\boldsymbol{e d}$ are located closely compared with the initial direction. Figures 8 and 9 show that the NSCP is very close to the design point, which is easier to find the design point than SCP at the mean values $\boldsymbol{x}_{\mathrm{m}}$.

The appropriate factor $f^{k}$ can be obtained with the method in Section 3.2 for each iteration. The dynamic factor vector $f^{1}$ obtained by the method in Section 3.2 for the first iteration is [1.1268 3.0000] and the sample points obtained with $f^{1}$ is shown in Figure 9, from which it can be seen that the sample points are closer to the LSF compared with the classical RSM. For this example, the center point for the third iteration is very close to the design point, which is shown in Figure 10. So the value of $f^{3}$ obtained with the method in

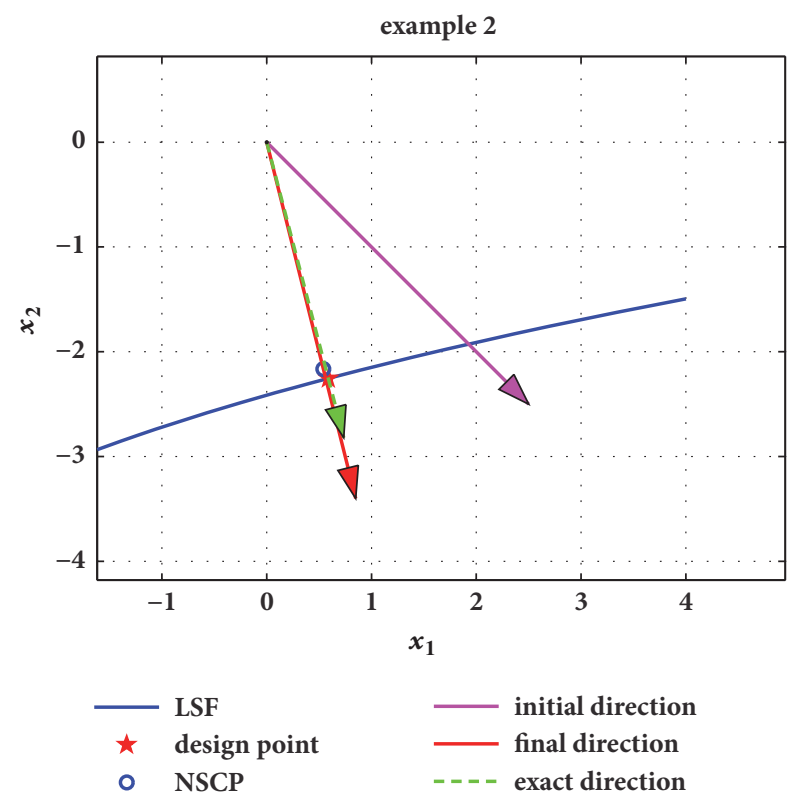

FIGURE 8: The new starting center point of example 2.

Section 3.2 is relative small so the details around the design point is obtained, which can make a precise result.

The final result of NDIRSM proposed in this paper is shown in Table 3. The algorithm is converged in 3 iterations with 21 sample points. The result of FORM is considered as the exact result. It can be seen from Table 3 that NDIRSM can achieve a more accurate solution with less sample points that represents the efficiency of the algorithm.

The processes of iteration are listed in Table 4 including the NDIRSM and the classical RSM with different $f^{k}$. NDIRSM is converged after three iterations, which is fewer than the classical RSM. Compared with the classical RSM, an accurate reliability index in the first iteration is obtained through the proposed RSM, which leads to a fewer iterations. So NDIRSM improves the computational efficiency and the result obtained by NDIRSM is more accurate compared with the other results in Table 3.

For this problem, only 5 sample points are required for each iteration and 7 sample points are required to obtain the NSCP, which lead to that the NDIRSM has not improved the efficiency significantly compared with the classical RSM with 
TABLE 4: Successive results of example 2 with different methods.

\begin{tabular}{|c|c|c|c|c|c|}
\hline \multirow{2}{*}{ items } & \multirow{2}{*}{$\beta$ (NDIRSM) } & \multicolumn{4}{|c|}{$\beta$ (classical RSM) } \\
\hline & & $f^{k}=3$ & $f^{k}=2$ & $f^{k}=1$ & $f^{k}=0.1$ \\
\hline First iteration & 2.3068 & \multirow{6}{*}{ Not convergent } & 2.6436 & 3.0522 & 3.1888 \\
\hline Final result & 2.3309 & & 2.3527 & 2.3332 & 2.3309 \\
\hline Number of sample points & 21 & & 83 & 29 & 23 \\
\hline Number of iteration & 3 & & 14 & 5 & 4 \\
\hline$x_{1}$ & 0.5860 & & 0.2898 & 0.5080 & 0.5937 \\
\hline$x_{2}$ & -2.2560 & & -2.3348 & -2.2773 & -2.2540 \\
\hline
\end{tabular}

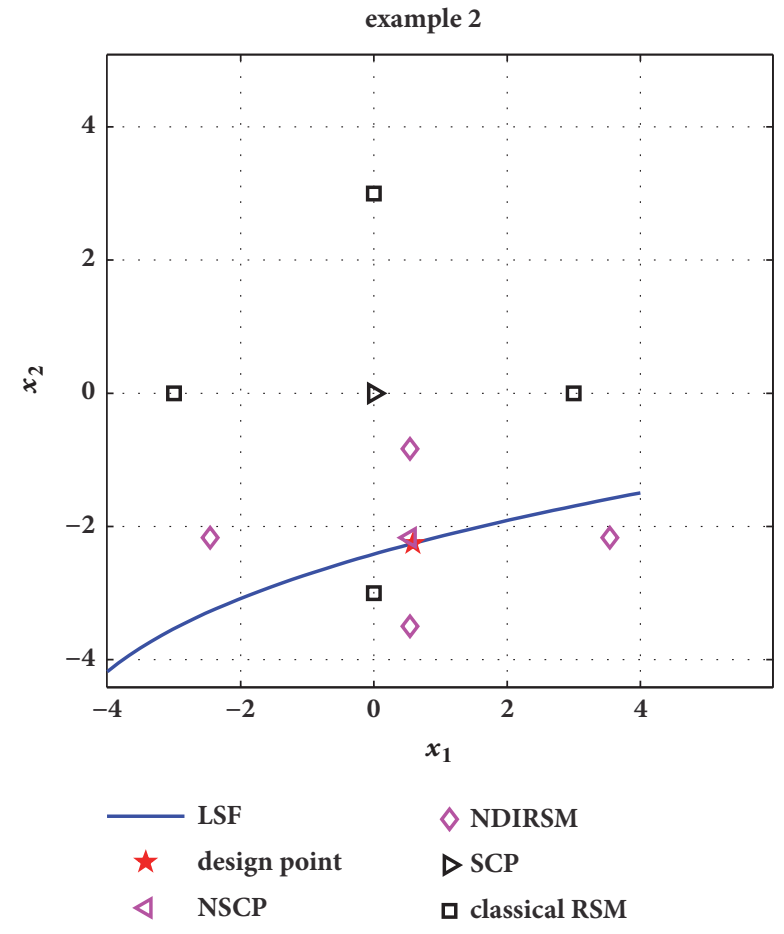

FIgURE 9: Sample points for the first iteration of example 2.

$f^{k}=0.1$. However, there are many input variables for practical engineering problems. So many sample points are required to obtain the result for each iteration, which is more than the sample points required to obtain NSCP. Thus NDIRSM can improve the efficiency significantly.

The final direction vector $\boldsymbol{u}$ of this example is $[0.5,-2]$. Then the exact direction vector $\boldsymbol{e d}$ obtained with the reference result listed in Table 3 is transformed with the same method as example 1 and the result is [0.521,-2.000]. It can be seen that the values of the corresponding components $u_{i}$ and $e d_{i}$ are almost the same and the directions they represent are close to each other as shown in Figure 8.

4.3. A Summarize. The two examples above illustrate the computational accuracy and efficiency of NDIRSM. The results of classical RSM listed in Tables 2 and 4 show that the values of arbitrary factor $f^{k}$ have a great influence on the computational accuracy and efficiency. If the center point around the design point is found, a small value of arbitrary factor $f^{k}$ will benefit the accuracy of reliability index on the

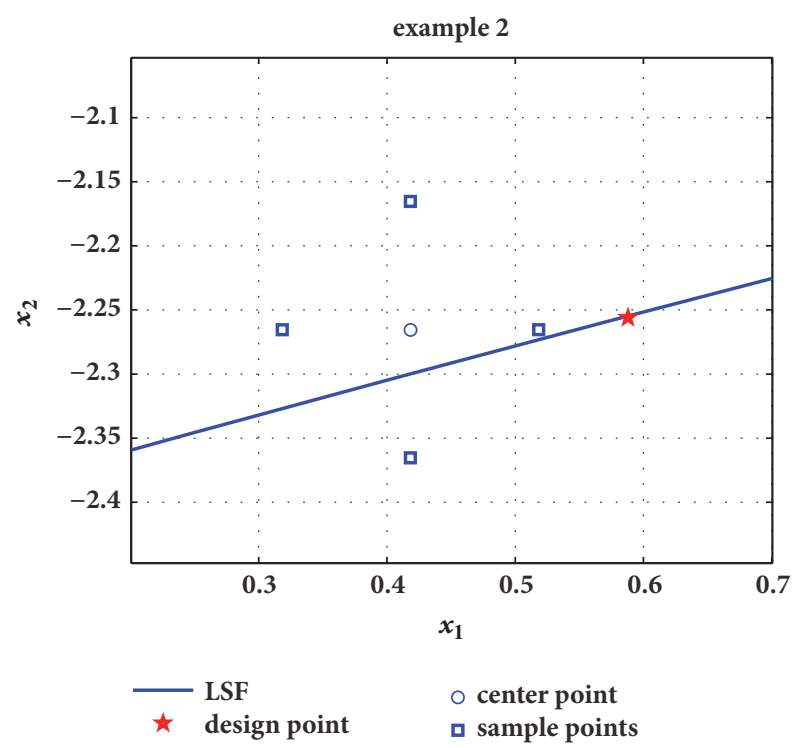

Figure 10: Sample points for the third iteration of example 2.

condition of the design matrix condition number of (3) not being ill-conditioned, which can also improve the efficiency. It can be concluded from Tables 1 and 3 that a SCP close to LSF is also helpful to improve the computational accuracy and efficiency.

The NDIRSM proposed in this paper calculates the NSCP and dynamic factor vector for the first iteration, which makes the sample points very close to the LSF around design point. Then in Section 3.2, the values of arbitrary factor $f^{k}$ are selected according to the design matrix condition number of (3) and an appropriate value is obtained. The results listed in Tables 1 and 3 show that NDIRSM improves the computational accuracy and efficiency of RSM.

\section{Probabilistic Analyses on Low Cycle Fatigue Life of Gas Turbine Disc}

Gas turbine disc is a critical component of an aero-engine. The large centrifugal loading and temperature gradient make the gas turbine disc suffering a significant tensile stress, which leads to low cycle fatigue (LCF) being one of the main failure models [29]. At the same time, there are many uncertain variables, such as the gas turbine geometry parameters, loadings, and material properties, which influence the LCF 


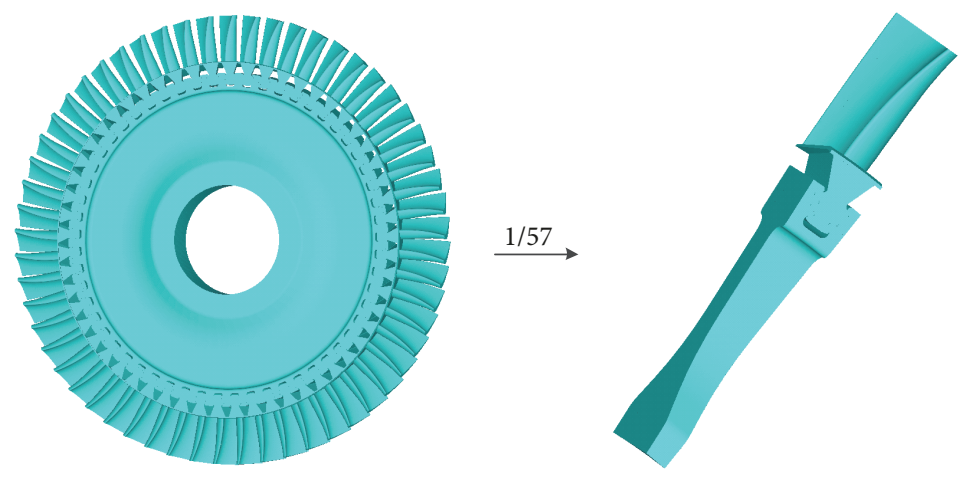

FIGURE 11: The geometric model of gas turbine disc with blades.

life of gas turbine disc, so the LCF life of gas turbine disc shows a significant scattering in nature [30-34]. Thus, it is necessary to analyse the failure probability of LCF life of gas turbine disc to reduce the risk. Besides, the input variables make it very complicated and time consuming to analyse the LCF life of gas turbine disc, especially for the reliability analyses. This section NDIRSM is applied to complete the reliability analyses on LCF life of gas turbine disc.

5.1. Gas Turbine Disc Model and Input Variables. A simplified model of gas turbine disc with blades, which is made of GH4169 superalloy, is selected as the study object. The turbine disc with blades is a cyclic symmetry structure. So a sector of the gas turbine disc with blade, which is 1/57 gas turbine disc, is created and the geometric model is shown in Figure 11. An appropriate symmetry boundary constraint is imposed on the sector disc. A general surface-to-surface contact is defined to represent the contact interaction between the blade and disc, which is a highly nonlinear behavior and requires larger computing resources. So it is very time consuming to perform reliability analysis on the turbine disc with blade. In order to improve the computational accuracy and efficiency, the NDIRSM proposed in this paper is used.

In the reliability analysis of the gas turbine disc with blades, there are many input random variables: $T_{1}$ represents the temperature on the upper of blade leading edge; $T_{2}$ represents the temperature on the lower of blade leading edge; $T_{3}$ represents the temperature on the upper of blade trailing edge; $T_{4}$ represents the temperature on the lower of blade trailing edge; $T_{5}$ represents the temperature on the root of the tenon; $T_{6}$ represents the temperature on the rim of the disc; $T_{7}$ represents the temperature on the lowest edge of the disc; $w$ represents the angular velocity; $E_{1}$ and $E_{2}$ represent the Young's modulus of the blade and disc, respectively; $u_{1}$ and $u_{2}$ represent the Poisson ratio of the blade and disc, respectively; $p_{1}$ and $p_{2}$ represent the density of the blade and disc, respectively; $a_{1}$ and $a_{2}$ represent the expansion coefficient of the blade and disc, respectively. The values and properties of them are listed in Table 5. So the input random vector is defined as

$$
\boldsymbol{x}=\left[E_{1}, E_{2}, u_{1}, u_{2}, p_{1}, p_{2}, a_{1}, a_{2}, w, T_{1}, T_{2}, T_{3}, T_{4}, T_{5}, T_{6}, T_{7}\right]
$$

5.2. Low Cycle Fatigue Life of Gas Turbine Disc. The finite element model and the result of the finite element execution are shown in Figure 12. It can be seen that the maximum stress is located in the corner of the third lower slot of the fir-tree rim region. So the LCF life in this location determines the LCF life of the gas turbine disc and the reliability analysis on the gas turbine disc LCF life is focused on the fir-tree rim region.

In order to predict the LCF life of the gas turbine disc, the Morrow formula $[35,36]$, which is an improvement of the Manson-Coffin $[37,38]$ formula by taking the mean stress into consideration, is employed. It can be expressed as

$$
\frac{\Delta \varepsilon_{\mathrm{t}}}{2}=\frac{\sigma_{\mathrm{f}}^{\prime}-\sigma_{\mathrm{m}}}{E}\left(2 N_{\mathrm{f}}\right)^{b}+\varepsilon_{\mathrm{f}}^{\prime}\left(2 N_{\mathrm{f}}\right)^{c}
$$

where $\Delta \varepsilon_{\mathrm{t}}$ is the strain range; the $\sigma_{\mathrm{f}}^{\prime}$ is the is the fatigue strength coefficient; $\sigma_{\mathrm{m}}$ is the mean strain; $\varepsilon_{\mathrm{f}}^{\prime}$ is the fatigue plasticity coefficient; $b$ is the fatigue strength exponent; $c$ is the fatigue plasticity exponent and $N_{\mathrm{f}}$ is the structural LCF life. Then the LCF life of gas turbine disc can be obtained with (18).

\subsection{Probabilistic Analysis on Low Cycle Fatigue Life of \\ Gas Turbine Disc}

5.3.1. Obtain the Allowed LCF Life of the Gas Turbine Disc according to Reliability Probability. After the LCF life of the gas turbine disc $N_{\mathrm{f}}(\boldsymbol{x})$ is calculated out with (18), the PF of the gas turbine disc LCF life can be expressed as

$$
g(\boldsymbol{x})=N_{\mathrm{f}}(\boldsymbol{x})-N_{\text {allow }}
$$

$N_{\text {allow }}$ denotes the allowed LCF life of the gas turbine disc.

The reliability probability value of the LCF life of gas turbine disc should be greater than or equal to $99.87 \%$ and the corresponding reliability index is 3.0115 which is obtained by $p_{f}=\Phi(-\beta)$. In order to get the value of $N_{\text {allow }}$ and analysis the relationship between the reliability index and $N_{\text {allow }}$, the reliability index is calculated out when $N_{\text {allow }}$ is equal to 1000 , $1500,1750,2000,2250,2500,2750,3000,3500$ and 4000, and the reliability index is obtained through NDIRSM.

The variation of the reliability probability with the $N_{\text {allow }}$ is presented in Figure 13(a). It can be seen that the reliability 
TABLE 5: Random properties.

\begin{tabular}{cccc}
\hline Random variables & Distribution type & Mean $($ unit $)$ & Standard division \\
\hline Material properties: & & & \\
$E_{1}$ & normal & $152(\mathrm{GPa})$ & $168.5(\mathrm{GPa})$ \\
$E_{2}$ & normal & 0.32 & 2100 \\
$u_{1}$ & normal & 0.32 & 2100 \\
$u_{2}$ & normal & $8.24 \times 10^{3}\left(\mathrm{Kg} / \mathrm{m}^{2}\right)$ & 100 \\
$p_{1}$ & normal & $8.24 \times 10^{3}\left(\mathrm{Kg} / \mathrm{m}^{2}\right)$ & 750 \\
$p_{2}$ & normal & $1.48 \times 10^{-5}\left({ }^{0} \mathrm{C}^{-1}\right)$ & 750 \\
$a_{1}$ & normal & $1.48 \times 10^{-5}\left({ }^{0} \mathrm{C}^{-1}\right)$ & 750 \\
$a_{2}$ & normal & & 750 \\
Multi-physical loads: & & $1357.168(\mathrm{rad} / \mathrm{s})$ & 13.57168 \\
$w$ & normal & $803\left({ }^{0} \mathrm{C}\right)$ & 8.03 \\
$T_{1}$ & normal & $705\left({ }^{0} \mathrm{C}\right)$ & 7.05 \\
$T_{2}$ & normal & $765\left({ }^{0} \mathrm{C}\right)$ & 7.65 \\
$T_{3}$ & normal & $670\left({ }^{0} \mathrm{C}\right)$ & 6.70 \\
$T_{4}$ & normal & $500\left({ }^{0} \mathrm{C}\right)$ & 5.00 \\
$T_{5}$ & normal & $625\left({ }^{0} \mathrm{C}\right)$ & 3.25 \\
$T_{6}$ & normal & $320\left({ }^{0} \mathrm{C}\right)$ & 3.20 \\
$T_{7}$ & normal & & \\
\hline
\end{tabular}
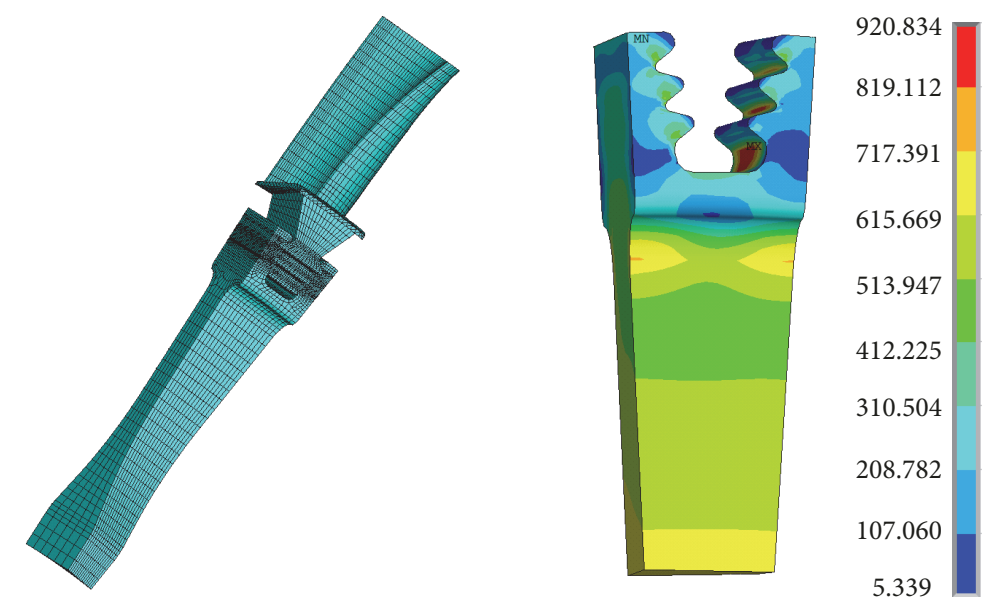

Figure 12: The finite element model and magnified Nodal von Mises equivalent stress distribution.

probability decreases with the increase of $N_{\text {allow }}$ and the LCF life is around 2000 when the reliability probability is equal to $99.87 \%$. In order to get an accurate allowed LCF life $N_{\text {allow, }}$, the data of the reliability index and the logarithm of LCF life of the gas turbine disc is fitted with a quadratic polynomial presented in Figure 13(b), according to which the allowed LCF life $N_{\text {allow }}$ is 1943.94 when reliability index is equal to 3.0115. The allowed LCF life is an integer, so the allowed LCF life $N_{\text {allow }}$ should be equal to 1943 to ensure the reliability.

5.3.2. Reliability Analysis. After the PF is obtained, reliability analyses on LCF life of gas turbine disc can be performed with NDIRSM. The processes of calculating the reliability index are listed in Table 6 including the NDIRSM and classical RSM with different $f^{k}$ when the allowed LCF life $N_{\text {allow }}$ is 1943 . NDIRSM is converged in three iterations with
117 finite element method executions, which is fewer than the classical RSM. It indicates that NDIRSM has a high efficiency.

According to the results listed in Tables 2 and 4, the reliability index obtained with small values of $f^{k}$ is very close to the exact reliability index. The results listed in Table 6 show that the reliability index calculated out with NDIRSM and the classical RSM are equal with each other approximately, which proves the correctness of the proposed RSM. The values of the PF with respect to the design point calculated out with different methods are listed in Table 6. It can be seen that the value of the PF at design point calculated out with NDIRSM is closer to zero than classical RSM. Since the design point is a point on the LSF, the value of the LSF at the exact design point should be equal to zero. So the value of PF at design point can be applied to illustrate the accuracy of the RSM and 


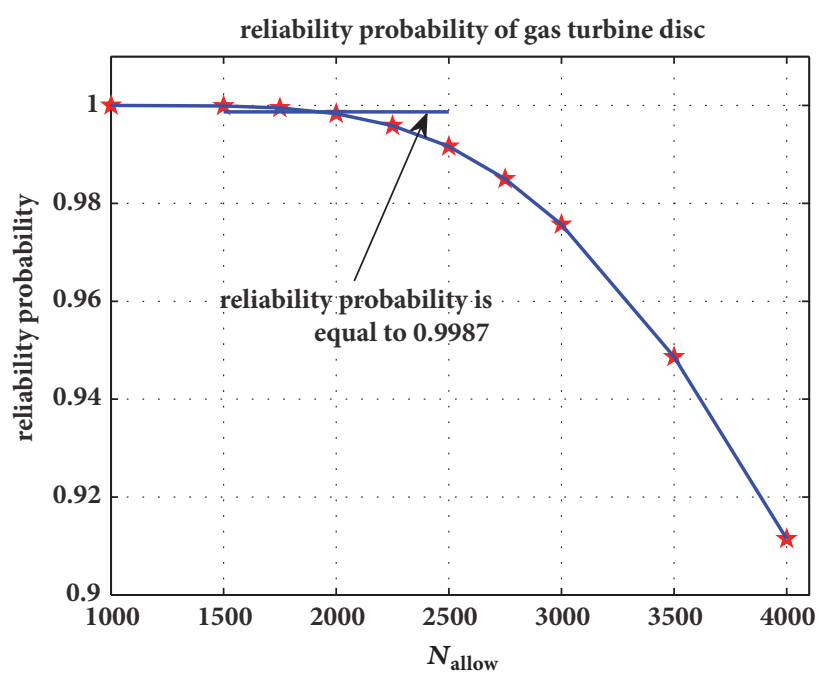

(a) Relationship between $N_{\text {allow }}$ and reliability probability of the gas turbine disc

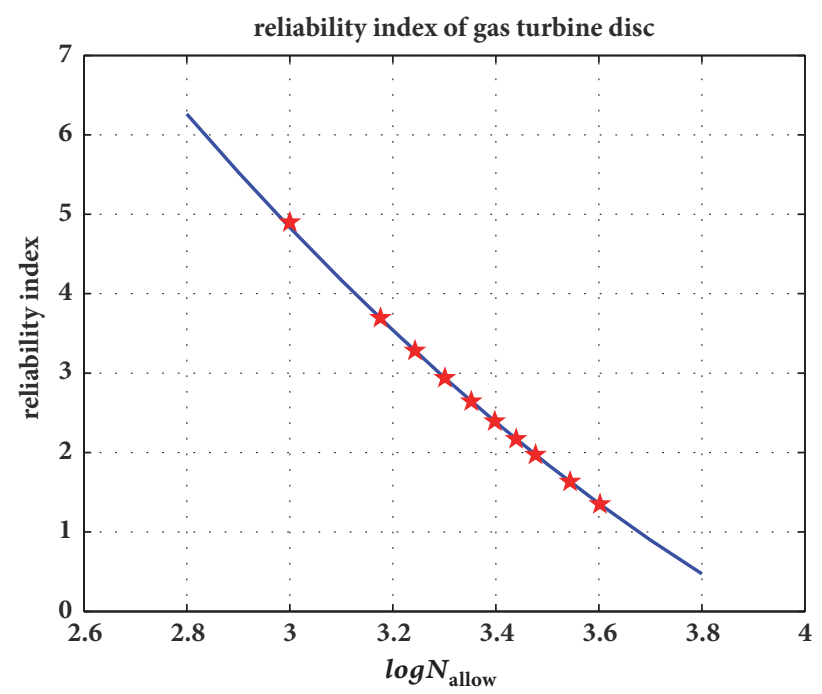

(b) Relationship between the logarithm of $N_{\text {allow }}$ and reliability index of the gas turbine disc

FIGURE 13: Reliability probability of gas turbine disc.

TABLE 6: Successive results.

\begin{tabular}{lccc}
\hline items & $\beta$ (NDIRSM) & $f^{k}=3$ & \multicolumn{2}{c}{$\beta$ (classical RSM) } & $f^{k}=2$ \\
& & 1.8607 & 1.8883 \\
First iteration & 3.0406 & 3.0102 & 3.0051 \\
Final result & 3.0036 & 4 & 5 \\
Number of iterations & 3 & 135 & 3.0027 \\
Number of sample points & 117 & -2.4131 & 4 \\
Value of PF at design point & -0.2325 & -1.2155 & 135 \\
\hline
\end{tabular}

the value of $\mathrm{PF}$ at design point listed in Table 6 demonstrates the accuracy of the result obtained by NDIRSM.

Some primary components of the final direction vector $\boldsymbol{u}$ are listed in Table 7 and the corresponding components of exact direction vector $\left(\boldsymbol{x}_{\mathrm{d}}-\boldsymbol{x}_{\mathrm{m}}\right)$./ $\boldsymbol{\sigma}$ ed are obtained with RSM3. Then ed is transformed with the same method as example 1 and the results are listed in Table 7 . It can be seen that the most values of the corresponding components $u_{i}$ and $e d_{i}$ are close to each other, which means that the directions they represent are located closely to each other.

\section{Conclusions}

The aim of this study is to develop an accurate and efficient reliability analysis method and a modified RSM called as NSIRSM for reliability analysis is proposed in this paper. For the first iteration, a NSCP, which is very close to the design point, is calculated out as the SCP; a dynamic factor vector is used to make the surrounding sample points close to LSF. For the subsequent iteration, the center point is the design point calculated out in the previous iteration and reasonable arbitrary factors are obtained according to the design matrix condition number, which makes the surrounding sample points close to LSF. Thus the algorithm can improve the accuracy and get a fast convergence. Several examples are employed in Section 4 to demonstrate the advantages of NDIRSM and the results show that NDIRSM improves the computational accuracy and efficiency of RSM. The comparison of the results of the classical RSM and NDIRSM indicates that NDIRSM can obtain a good result after the first iteration. The results obtained by the classical RSM with different arbitrary factors illustrate that the value of the arbitrary factor has a great influence on the computational accuracy and efficiency of RSM. A small value of the arbitrary factor will benefit the computational accuracy and efficiency if a center point around the design point is found.

Then NDIRSM is applied to analyse the reliability probability of the LCF life of the gas turbine disc. The reliability probability of the LCF life of the gas turbine disc is calculated out with the classical RSM and NDIRSM. The comparison of them illustrates the efficiency and correctness of NDIRSM and the values of $\mathrm{PF}$ at design point demonstrate the accuracy of NDIRSM. The relationship between the logarithm of LCF life of gas turbine disc and the reliability index is obtained, according to which the allowed LCF life of the gas turbine disc can be got when the reliability probability is greater than or equal to a threshold value. This provides a useful reference for reliability analysis on low cycle fatigue life of gas turbine disc and enriches the theory of mechanical design. 
TABLE 7: Some components of exact direction vector $\boldsymbol{e d}$ and final direction $\boldsymbol{u}$.

\begin{tabular}{lcccccccccc}
\hline variables & $E_{1}$ & $p_{1}$ & $a_{1}$ & $E_{2}$ & $u_{2}$ & $p_{2}$ & $a_{2}$ & $w$ & $T_{5}$ & $T_{6}$ \\
\hline$e d_{i}$ & 0.130 & 1.731 & -1.458 & -0.287 & -0.131 & 0.251 & 1.370 & 2.000 & 0.124 & -0.973 \\
$u_{i}$ & 0.500 & 2.000 & -1.000 & -0.500 & -0.500 & 0.500 & 0.500 & 2.000 & 0.500 & -0.500 \\
\hline
\end{tabular}

\section{Data Availability}

The nonconfidential data are available from the corresponding author upon reasonable request.

\section{Conflicts of Interest}

The authors declare that there are no conflicts of interest regarding the publication of this paper.

\section{Acknowledgments}

This research was supported by the National Natural Science Foundation of China (Grant nos. 51335003 and 11772011).

\section{References}

[1] P. Bjerager, "Probability integration by directional simulation," Journal of Engineering Mechanics, vol. 114, no. 8, pp. 1285-1302, 1988.

[2] A. M. Hasofer and N. C. Lind, "Exact and invariant secondmoment code format(for reliability analysis in multivariate problems," American Society of Civil Engineers, Engineering Mechanics Division, Journal, vol. 100, pp. 111-121, 1974.

[3] R. Rackwitz and B. Flessler, "Structural reliability under combined random load sequences," Computers \& Structures, vol. 9, no. 5, pp. 489-494, 1978.

[4] A. Der Kiureghian, H.-Z. Lin, and S.-J. Hwang, "Second-order reliability approximations," Journal of Engineering Mechanics, vol. 113, no. 8, pp. 1208-1225, 1987.

[5] P.-L. Liu and A. der Kiureghian, "Optimization algorithms for structural reliability," Structural Safety, vol. 9, no. 3, pp. 161-177, 1991.

[6] C. G. Bucher and U. Bourgund, "A fast and efficient response surface approach for structural reliability problems," Structural Safety, vol. 7, no. 1, pp. 57-66, 1990.

[7] M. R. Rajashekhar and B. R. Ellingwood, "A new look at the response surface approach for reliability analysis," Structural Safety, vol. 12, no. 3, pp. 205-220, 1993.

[8] S.-H. Kim and S.-W. Na, "Response surface method using vector projected sampling points," Structural Safety, vol. 19, no. 1, pp. 3-19, 1997.

[9] N. Gayton, J. M. Bourinet, and M. Lemaire, "CQ2RS: A new statistical approach to the response surface method for reliability analysis," Structural Safety, vol. 25, no. 1, pp. 99-121, 2003.

[10] I. Kaymaz and C. A. McMahon, "A response surface method based on weighted regression for structural reliability analysis," Probabilistic Engineering Mechanics, vol. 20, no. 1, pp. 11-17, 2005.

[11] X. S. Nguyen, A. Sellier, F. Duprat, and G. Pons, "Adaptive response surface method based on a double weighted regression technique," Probabilistic Engineering Mechanics, vol. 24, no. 2, pp. 135-143, 2009.
[12] S.-C. Kang, H.-M. Koh, and J. F. Choo, "An efficient response surface method using moving least squares approximation for structural reliability analysis," Probabilistic Engineering Mechanics, vol. 25, no. 4, pp. 365-371, 2010.

[13] D. L. Allaix and V. I. Carbone, "An improvement of the response surface method," Structural Safety, vol. 33, no. 2, pp. 165-172, 2011.

[14] X. L. Guan and R. E. Melchers, "Effect of response surface parameter variation on structural reliability estimates," Structural Safety, vol. 23, no. 4, pp. 429-444, 2001.

[15] H. P. Gavin and S. C. Yau, "High-order limit state functions in the response surface method for structural reliability analysis," Structural Safety, vol. 30, no. 2, pp. 162-179, 2008.

[16] N. Roussouly, F. Petitjean, and M. Salaun, "A new adaptive response surface method for reliability analysis," Probabilistic Engineering Mechanics, vol. 32, pp. 103-115, 2013.

[17] B. Gaspar, A. P. Teixeira, and C. G. Soares, "Assessment of the efficiency of Kriging surrogate models for structural reliability analysis," Probabilistic Engineering Mechanics, vol. 37, pp. 24-34, 2014.

[18] S. Goswami, S. Ghosh, and S. Chakraborty, "Reliability analysis of structures by iterative improved response surface method," Structural Safety, vol. 60, pp. 56-66, 2016.

[19] M. A. Shayanfar, M. A. Barkhordari, and M. A. Roudak, "An efficient reliability algorithm for locating design point using the combination of importance sampling concepts and response surface method," Communications in Nonlinear Science and Numerical Simulation, vol. 47, pp. 223-237, 2017.

[20] A. Hadidi, B. F. Azar, and A. Rafiee, "Efficient response surface method for high-dimensional structural reliability analysis," Structural Safety, vol. 68, pp. 15-27, 2017.

[21] J. Cheng, Q. S. Li, and R.-C. Xiao, "A new artificial neural network-based response surface method for structural reliability analysis," Probabilistic Engineering Mechanics, vol. 23, no. 1, pp. 51-63, 2008.

[22] J. Deng, D. Gu, X. Li, and Z. Q. Yue, "Structural reliability analysis for implicit performance functions using artificial neural network," Structural Safety, vol. 27, no. 1, pp. 25-48, 2005.

[23] A. H. Elhewy, E. Mesbahi, and Y. Pu, "Reliability analysis of structures using neural network method," Probabilistic Engineering Mechanics, vol. 21, no. 1, pp. 44-53, 2006.

[24] B. Richard, C. Cremona, and L. Adelaide, "A response surface method based on support vector machines trained with an adaptive experimental design," Structural Safety, vol. 39, pp. 1421, 2012.

[25] X. Li, X. Li, and Y. Su, "A hybrid approach combining uniform design and support vector machine to probabilistic tunnel stability assessment," Structural Safety, vol. 61, pp. 22-42, 2016.

[26] U. Alibrandi, A. M. Alani, and G. Ricciardi, "A new sampling strategy for SVM-based response surface for structural reliability analysis," Probabilistic Engineering Mechanics, vol. 41, pp. 112, 2015.

[27] C. Elegbede, "Structural reliability assessment based on particles swarm optimization," Structural Safety, vol. 27, no. 2, pp. 171-186, 2005. 
[28] F. Duprat and A. Sellier, "Probabilistic approach to corrosion risk due to carbonation via an adaptive response surface method," Probabilistic Engineering Mechanics, vol. 21, no. 3, pp. 207-216, 2006.

[29] R. Wang, X. Liu, D. Hu, F. Meng, D. Li, and B. Li, "Zonebased reliability analysis on fatigue life of GH720Li turbine disk concerning uncertainty quantification," Aerospace Science and Technology, vol. 70, pp. 300-309, 2017.

[30] H. Gao, C. Fei, G. Bai, and L. Ding, "Reliability-based lowcycle fatigue damage analysis for turbine blade with thermostructural interaction," Aerospace Science and Technology, vol. 49, pp. 289-300, 2016.

[31] R. Leverant Gerald, R. C. McClung, Y.-T. Wu et al., “Turbine rotor material design," No. TR-18-7445, Southwest Research Institute, San Antonio, TX, USA, 2000.

[32] Z. Shun-Peng, H.-Z. Huang, W. Peng et al., "Probabilistic physics of failure-based framework for fatigue life prediction of aircraft gas turbine discs under uncertainty," Reliability Engineering \& System Safety, vol. 146, pp. 1-12, 2016.

[33] L.-K. Song, J. Wen, C.-W. Fei, and G.-C. Bai, "Distributed collaborative probabilistic design of multi-failure structure with fluid-structure interaction using fuzzy neural network of regression," Mechanical Systems and Signal Processing, vol. 104, pp. 72-86, 2018.

[34] J. Mao, D. Hu, D. Li, R. Wang, and J. Song, "Novel adaptive surrogate model based on LRPIM for probabilistic analysis of turbine disc," Aerospace Science and Technology, vol. 70, pp. 7687, 2017.

[35] J. D. Morrow, "Cyclic plastic strain energy and the fatigue of metals," Internal Friction, Damping, and Cyclic Plasticity, ASTM Spec. Tech. Publ, vol. 378, 1965.

[36] C. L. Liu, Z. Z. Lu, Y. L. Xu, and Z. F. Yue, "Reliability analysis for low cycle fatigue life of the aeronautical engine turbine disc structure under random environment," Materials Science and Engineering: A Structural Materials: Properties, Microstructure and Processing, vol. 395, no. 1-2, pp. 218-225, 2005.

[37] S. S. Manson, "Behavior of materials under conditions of thermal stress," National Advisory Commission on Aeronautics. Report 1170, Lewis Flight Propulsion Laboratory, Cleveland, OH, USA, 1954.

[38] L. F. Coffin, "A study of the effects of cyclic thermal stress on a ductile metal," Transactions of the American Society of Mechanical Engineers, vol. 76, pp. 931-950, 1954. 


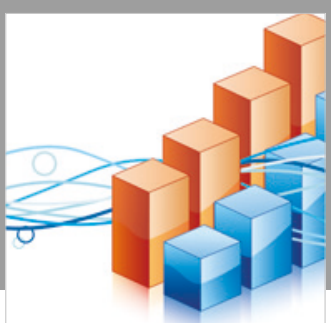

Advances in

Operations Research

\section{-n-m}
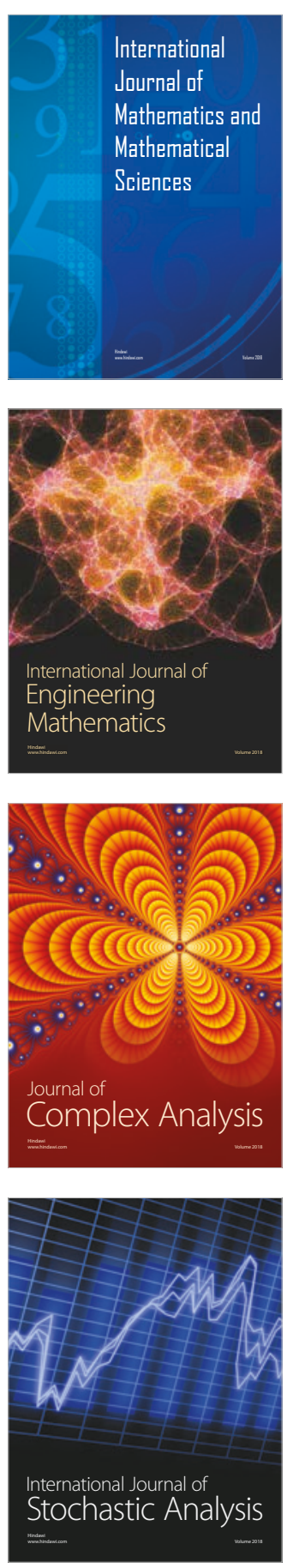
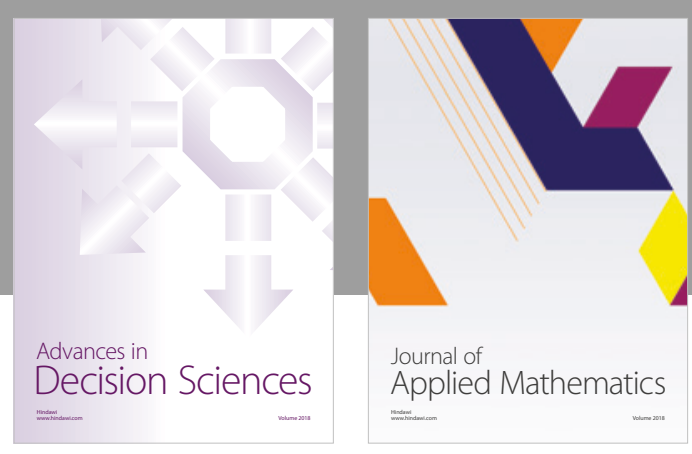

Journal of

Applied Mathematics
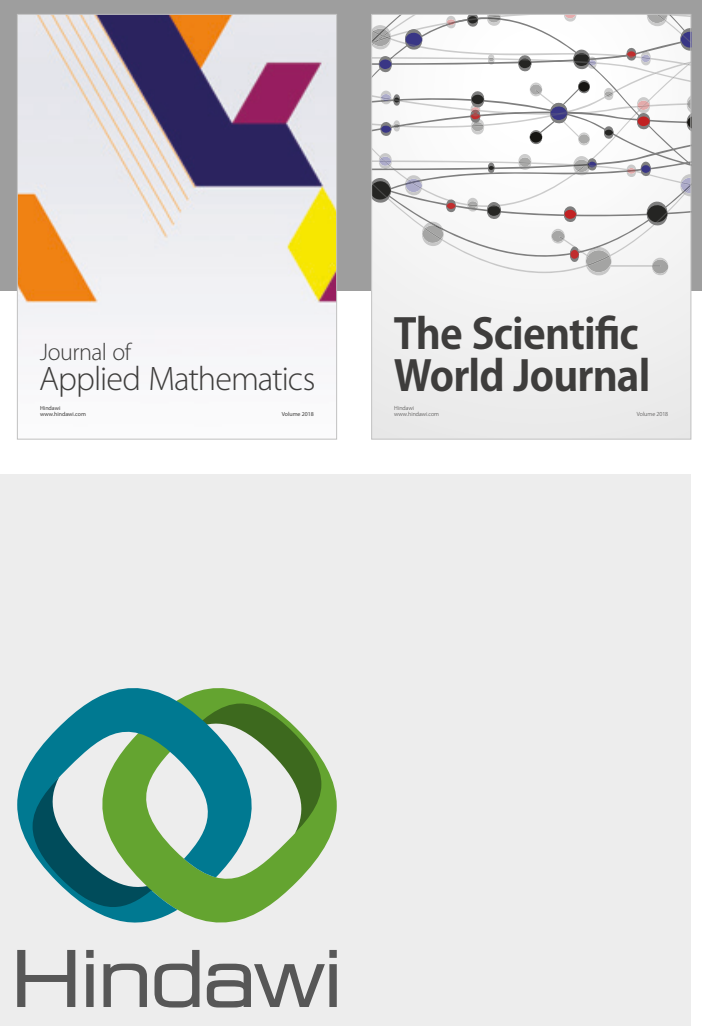

Submit your manuscripts at

www.hindawi.com

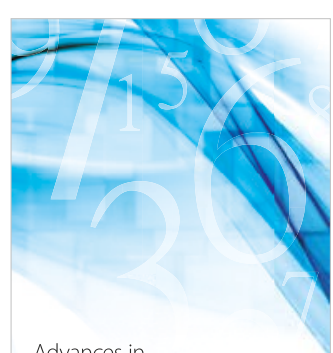

Advances in
Numerical Analysis
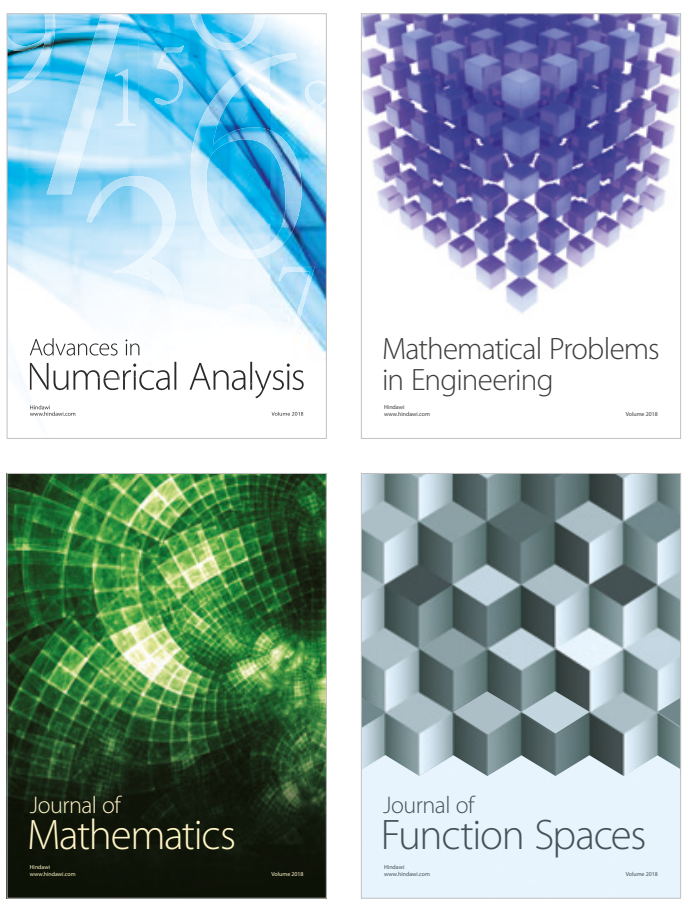

Mathematical Problems in Engineering

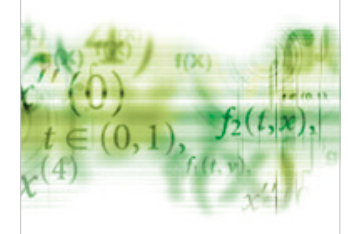

International Journal of

Differential Equations

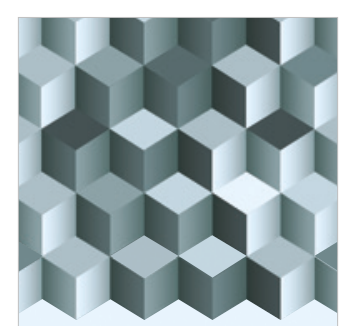

Journal of

Function Spaces

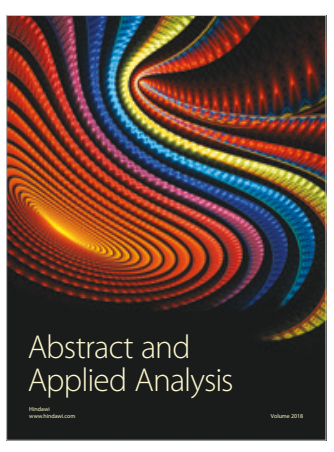

The Scientific

World Journal

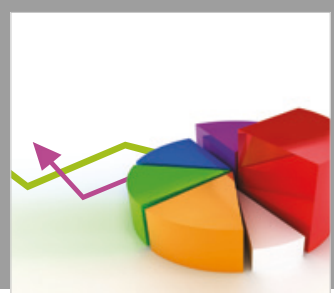

Journal of

Probability and Statistics
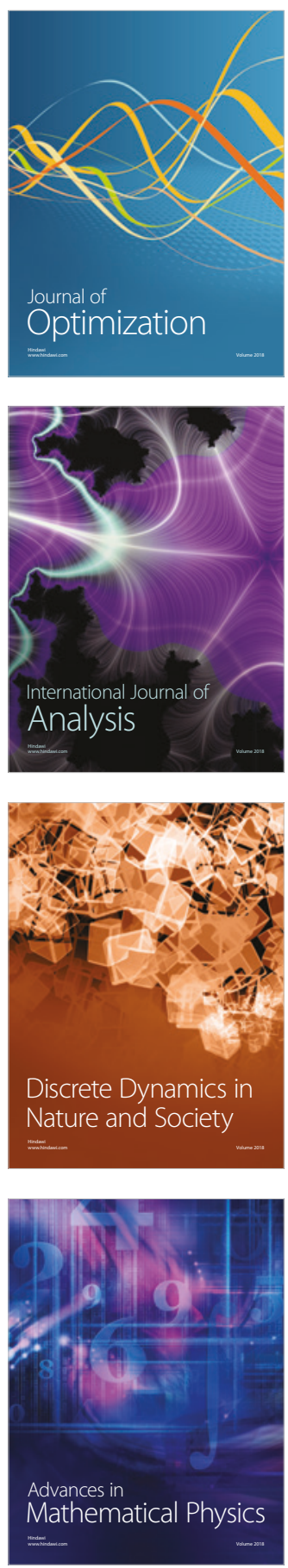